\title{
Dickkopf-3 upregulation mediates the cardioprotective effects of curcumin on chronic heart failure
}

\author{
QUAN CAO ${ }^{1-3}$, JUNXIA ZHANG ${ }^{4}$, LING GAO $^{5}$, YIJIE ZHANG ${ }^{1-3}$, MINGYAN DAI $^{1-3}$ and MINGWEI BAO ${ }^{1-3}$ \\ ${ }^{1}$ Department of Cardiology, Renmin Hospital of Wuhan University; ${ }^{2}$ Cardiovascular Research Institute, Wuhan University; \\ ${ }^{3}$ Hubei Key Laboratory of Cardiology, Wuhan, Hubei 430060; ${ }^{4}$ Department of Endocrinology, Wuhan General \\ Hospital of The Chinese People's Liberation Army, Wuhan, Hubei 430070; ${ }^{5}$ Department of Endocrinology \\ and Metabolism, Renmin Hospital of Wuhan University, Wuhan, Hubei 430060, P.R. China
}

Received October 7, 2017; Accepted February 28, 2018

DOI: $10.3892 / \mathrm{mmr} .2018 .8783$

\begin{abstract}
Curcumin, isolated from rhizome of turmeric, has been widely studied as a potential therapeutic drug for cancer. However, protective effects of curcumin on chronic heart failure (CHF) have not been fully studied. In the present study, the effects of curcumin on $\mathrm{CHF}$ and the underlying mechanisms were investigated. A total of 40 rabbits were randomized into 4 groups: Control rabbits fed with placebo (Con) or curcumin (Con-cur), CHF rabbits fed with placebo (CHF) or curcumin (CHF-cur). CHF was induced by volume and pressure overload. The effects of curcumin on cardiac function and left ventricular (LV) structure were assessed by echocardiography and histology. The effects of curcumin on CHF molecular biomarkers were detected by dihydroethidium and immunohistochemical staining. The effects of curcumin on Dickkopf-related protein 3 (DKK-3), p38 mitogen-activated protein kinase (p38), c-Jun N-terminal kinase (JNK) and apoptosis signal-regulating kinase 1 (ASK1) were assessed by immunohistochemical staining and western blot analysis. Cardiac dysfunction and LV remodeling were successfully produced by ten weeks volume overload and eight weeks pressure overload in the CHF group. Compared with the Con group, the $\mathrm{CHF}$ group demonstrated higher levels of $\mathrm{CHF}$ molecular biomarkers, a lower level of DKK-3 expression and alterations of $\mathrm{p} 38$, JNK and ASK1 protein expression. Curcumin alleviated all those abnormalities markedly in the CHF-cur group. In summary, curcumin may exert cardioprotective effects by up-regulating DKK-3, which in turn may inhibit p38 and JNK signaling pathways in an ASK1-dependent way. The present study demonstrated that Dickkopf-3 upregulation mediates the
\end{abstract}

Correspondence to: Professor Mingwei Bao, Department of Cardiology, Renmin Hospital of Wuhan University, 238 Jiefang Road, Wuhan, Hubei 430060, P.R. China

E-mail:mbao@whu.edu.cn

Key words: curcumin, chronic heart failure, Dickkopf-related protein 3, remodeling cardioprotective effects of curcumin on chronic heart failure for the first time.

\section{Introduction}

With a growing epidemic, chronic heart failure (CHF) results in an economic burden worldwide and has become a major factor causing disabilities and mortality $(1,2)$. CHF is characterized by progressive cardiac dysfunction and remodeling $(3,4)$. Volume and pressure overload are the most common promoters in pathological process of CHF (5). Cellular and molecular mechanisms are involved in the deterioration of $\mathrm{CHF}$, including inflammation, oxidative stress and fibrosis but the underlying mechanisms are not fully understood (6).

Curcumin [1,7-bis (4-hydroxy-3-methoxyphenyl)-1,6-heptadiene-3,5-dione] is the major active component of turmeric isolated from the rhizome of Zingiberaceae and has widely been studied as an anticancer agent $(7,8)$. Curcumin is a powerful antioxidant and anti-inflammatory reagent (9), as well as a modulator of protein kinase, DNA methyltransferase and histone acetyltransferase (10). Curcumin is closely associated with the expression and activity of various regulatory proteins (11). Several studies have reported the protective effects of curcumin on cardiovascular diseases, including protection against cardiac dysfunction and remodeling in heart failure in rats $(8,12,13)$. However, the mechanism by which curcumin exerts cardioprotective effects has not been fully understood.

Dickkopf-related protein 3 (DKK-3), a member of the Dickkopf glycoprotein family, is recognized as a negative regulator of many tumors and is crucially involved in cell growth, proliferation and immunity (14). DKK-3 is abundantly expressed in skeletal muscle and heart $(15,16)$. DKK-3 expression was decreased in hypertrophic murine heart and human dilated cardiomyopathy heart (5). In animal models, cardiac dysfunction and remodeling induced by pressure overload can be exacerbated by DKK-3 depletion and can be rescued by DKK-3 overexpression (5). Our previous study indicated that DKK-3 served a cardioprotective role in the pathology of CHF by inhibiting p38 mitogen-activated protein kinase (p38) and c-JunN-terminal kinase (JNK) 
signaling pathways in an apoptosis signal-regulating kinase 1 (ASK1)-dependent way (16). DKK-3 may be the downstream target of curcumin owing to its similar cardioprotective effects in heart failure. The present study was designed to investigate whether curcumin exert cardiac protective effects by regulating DKK-3 and try to clarify whether p38 and JNK pathways are involved in the underlying molecular mechanisms.

\section{Materials and methods}

Animal preparation. A total of 40 adult New Zealand rabbits (male, weighing 2-2.5 kg, 12-week-old) were kept in the breeding facility for one week prior to experimental use. Rabbits were singly housed in animal holding cages at temperature $\left(22 \pm 2^{\circ} \mathrm{C}\right)$ and humidity $(45-50 \%)$, with a 12 -h light/dark cycle and unrestricted access to food and water. Rabbits were randomized into four groups: Control rabbits given with placebo (Con, $\mathrm{n}=10$ ) or curcumin (Con-cur, $\mathrm{n}=10$ ), chronic heart failure rabbits fed with placebo $(\mathrm{CHF}, \mathrm{n}=10)$ or curcumin (CHF-cur, $n=10)$. Placebo or curcumin were fed three days prior to the pressure overload surgery and this continued until the day of sacrifice. The dose of curcumin (Hebei Food Additive Co., Ltd. Shijiazhuang, Hebei, China) and placebo (Kangxin Co., Ltd., Shijiazhuang, Hebei, China) both were $100 \mathrm{mg} / \mathrm{day} / \mathrm{kg}$ in capsules, which were chosen with reference to a previous study (17). The placebo was made of starch and was contained in an identical capsule to that of curcumin. All procedures were according to the 'Guide for the Care and Use of Laboratory Animals' published by the US National Institutes of Health (NIH Publication no. 85-23; revised 1996) and approved by the Institutional Animal Care and Use Committee at Renmin Hospital of Wuhan University (Wuhan, China).

CHF production. CHF was induced by ten weeks volume overload and eight weeks pressure overload as previously described with slight modifications (18). Prior to each surgery, all rabbits were anesthetized with pentobarbital sodium (30 mg/kg) by intravenous injection. Volume overload was produced by puncturing across the aortic valve through carotid artery retrogradely with a catheter (external diameter $1.30 \mathrm{~mm}$ ). The catheter puncturing across the aortic valve was repeated until the pulse pressure increased by $\sim 100 \%$. Two weeks following this, pressure overload was produced by ligating the abdominal aorta partly just above renal artery branch out. This resulted in abdominal aorta stenosis by $\sim 50 \%$ in $\mathrm{CHF}$ rabbits. Rabbits in the Con and Con-cur groups underwent a sham operation, but neither aortic valve regurgitation nor aorta stenosis was produced.

Cardiac function and structure evaluation by echocardiography. Transthoracic echocardiography (10S transducer, Vivid 7, GE Healthcare, Chicago, IL, USA) was performed at baseline and eight weeks following the aorta stenosis surgery. Left ventricular ejection fraction (LVEF), left ventricular fractional shortening (LVFS), aortic diameter (AO), left ventricular posterior wall thickness (LVPW) and interventricular septal thickness (IVS) were assessed by echocardiography.
Heart weight and cardiac histology. Following the echocardiography, all rabbits were sacrificed and tissue samples from left ventricular (LV) were prepared for the following analysis. LV samples were fixed in $4 \%$ paraformaldehyde solution at room temperature for $24 \mathrm{~h}$ and then were sliced into $5-\mu \mathrm{m}$ sections transversely. Haematoxylin \& eosin (H\&E), masson (G1006, Servicebio, Inc., Wuhan, China), picrosirius red (PSR) (G1018, Servicebio, Inc.) staining and terminal deoxynucleotidyl transferase-mediated dUTP nick end labeling (TUNEL) assay (In Situ Cell Death Detection kit; cat. no. 11684817910; Roche Diagnostics, Basel, Switzerland) were performed. In $\mathrm{H} \& \mathrm{E}$ staining, the slides were successively stained with hematoxylin solution and eosin solution at room temperature for 5 min respectively. In masson staining, the slides were successively stained with iron hematoxylin solution, acid ponceau fuchsin solution and aniline blue solution at room temperature for $5 \mathrm{~min}$ each respectively. In PSR staining, the slides were stained with sirius red solution at room temperature for 10 min. In TUNEL staining, slides of LV were first incubated with the TUNEL reaction mixture containing terminal deoxynucleotidyl transferase (TdT) at $37^{\circ} \mathrm{C}$ for $60 \mathrm{~min}$. Subsequently, diaminobenzidine was added to the slides for $30 \mathrm{~min}$ and incubated in substrate solution for an additional $15 \mathrm{~min}$ at $37^{\circ} \mathrm{C}$. The cross-sectional area (CSA) of LV myocytes was analyzed based on H\&E staining, LV interstitial collagen volume (\%) was evaluated based on both Masson and PSR staining by Image-Pro Plus 6.0 software (Media Cybernetics, Inc., Rockville, MD, USA). The cardiomyocyte apoptosis index was defined as the proportion of apoptotic cardiomyocytes among the total cardiomyocytes (apoptotic cardiomyocytes had deep-brown nuclei while normal cardiomyocytes had blue nuclei).

Ultrastructure analysis. For cardiac ultrastructure analysis, LV samples were immediately soaked in pre-cooled $\mathrm{Ca}^{2+}$-free phosphate saline buffer (G1102; Servicebio, Inc.). The samples were successively fixed with $2.5 \%$ glutaraldehyde and $1 \%$ osmium tetroxide at $4{ }^{\circ} \mathrm{C}$ for $2 \mathrm{~h}$. Then the samples were dehydrated by ethanol of gradient dilution $(30,50$ and $70 \%$ ) at $4^{\circ} \mathrm{C}$ for $10 \mathrm{~min}$ respectively, later the samples were further dehydrated using an acetone of gradient dilution $(80,90$ and $100 \%)$ at room temperature for $10 \mathrm{~min}$. Samples were sliced into $50 \mathrm{~nm}$ section, embedded in the Epon resin and then stained with uranyl acetate and lead citrate at $40^{\circ} \mathrm{C}$ for $4 \mathrm{~h}$. Sections were imaged under a transmission electron microscope (HT7700; Hitachi, Ltd., Tokyo, Japan).

Inflammation, hypertrophy and fibrosis biomarkers analysis. Reactive oxygen species (ROS) production of LV myocytes was detected by dihydroethidium (DHE; $10 \mathrm{mmol} / \mathrm{l}$; D7008; Sigma-Aldrich; Mecrk KGaA, Darmstadt, Germany) staining. Cryosections $(10-\mu \mathrm{m})$ were treated for $30 \mathrm{~min}$ at $37^{\circ} \mathrm{C}$, and the ethidium fluorescence (excitation/emission at 488/610 nm) was examined by digital fluorescence microscopy (Eclipse Ti-SR; Nikon Corporation, Tokyo, Japan). The relative fluorescence intensity was analyzed by Image-Pro Plus 6.0 software (Media Cybernetics, Inc.). Immunohistochemical staining was performed as described previously (19). LV sections were incubated overnight at $4^{\circ} \mathrm{C}$ with primary antibodies respectively as follows: Mouse monoclonal anti-tumor necrosis 
factor-alpha (TNF- $\alpha$ ) antibody (1:200; cat. no. NB600-1422; Novus Biologicals, Ltd., Cambridge, UK), mouse monoclonal anti-matrix metallopeptidase-2 (MMP-2) antibody (1:200; cat. no. ab2462; Abcam, Cambridge, UK), mouse monoclonal anti-matrix metallopeptidase-9 (MMP-9) antibody (1:200; cat. no. ab58803; Abcam) and mouse monoclonal anti-sarcoplasmic reticulum $\mathrm{Ca}^{2+}$ ATPase (SERCA2a) antibody (1:200; cat. no. ab2817; Abcam). Following washes with PBS three times, sections were incubated with the rabbit anti-mouse immunoglobulin (Ig) M/horseradish peroxidase (HRP) secondary antibody (1:200; cat. no. bs-0368R-HRP; BIOSS, Beijing, China). A total of five fields of view (magnification, $\mathrm{x} 200$ ) were selected randomly for each slide under a light microscope (BX50, Olympus Corporation, Tokyo, Japan) and the relative mean optical density of ROS or each target protein was analyzed by Image-Pro Plus 6.0 software (Media Cybernetics, Inc.).

Detection of DKK-3, p38, JNK and ASK1 expression. Immunohistochemical staining for DKK-3 was performed as described above. Primary rabbit polyclonal anti-DKK-3 antibody (1:200; cat. on. bs-2686R; BIOSS) and secondary antibody mouse anti-rabbit IgM/HRP (1:200, bs-0369M-HRP, BIOSS) were used in the staining. Western blotting was performed by using extracts from LV tissue homogenates. Radio-immunoprecipitation assay lysis buffer (G2002 Servicebio, Inc.) was used for protein extraction. The protein concentration of the LV tissue homogenates was measured by Pierce BCA Protein Assay kit (cat. no. 23225; Pierce; Thermo Fisher Scientific, Inc. Waltham, MA, USA) according to the manufacturer's protocol following centrifugation $\left(4^{\circ} \mathrm{C}, 10 \mathrm{~min}\right.$, $15,000 \times \mathrm{g})$. SDS-PAGE gel wells were loaded with $50 \mu \mathrm{g}$ of corresponding protein for electrophoresis. The proteins were transferred to a polyvinylidene fluoride membrane. The blots were incubated with the primary antibodies overnight at $4^{\circ} \mathrm{C}$ as follows: Rabbit polyclonal anti-DKK-3 antibody (1:1,000; cat.no. bs-2686R), rabbit polyclonal anti-p38 antibody $(1: 1,000$; cat. no. bs-0637R), rabbit polyclonal anti-phospho-p38 (Thr180+Tyr182) antibody (p-p38; 1:1,000; cat. no. bs-0636R), rabbit polyclonal anti-JNK (1:1,000; cat. no. bs-10562R), rabbit anti-phospho-JNK (Thr183+Tyr185) antibody (P-JNK; 1:1,000; cat. no. bs-1640R), rabbit polyclonal anti-ASK1 (1:1,000; cat. no. bs-1425R), rabbit anti-phospho-ASK1 (Ser1033) antibody (P-ASK1; 1:1,000; cat. no. bs-5437R) and antibody against GAPDH (1:5,000; cat. no. bs-2188R). Subsequently, the membrane was incubated with the mouse anti-rabbit IgM/HRP secondary antibody (1:5,000; cat. no. bs-0369M-HRP) for $1 \mathrm{~h}$ at room temperature. All antibodies used in western blot analysis were purchased from BIOSS. Visualization reagent (ECL; cat. no. G2014; Servicebio, Inc.) was used in the western blotting. Membranes were analyzed by Quantity One Imaging system (version 4.6.9; Bio-Rad Laboratories, Inc., Hercules, CA, USA). The protein expression was normalized to that of GAPDH.

Statistical analysis. Quantitative data are presented as the mean \pm standard deviation. Comparison between preoperative and postoperative parameters was performed by matched Student's t-test. Multi-group comparisons were examined via one-way analysis of variance followed by a
Student-Newman-Keuls post hoc test. The statistical differences were examined by the statistical software SPSS package v22.0 (IBM Corp., Armonk, NY, USA). P<0.05 was considered to indicate a statistically significant difference.

\section{Results}

Curcumin improves cardiac function. The present study aimed to determine the effect of curcumin on chronic heart failure and whether curcumin improved cardiac function by observed symptoms and echocardiography. All rabbits suffered from evident dyspnea and edema in the CHF group, and six rabbits suffered from cough in the CHF-cur group. Based on the echocardiography data (Table I), LVEF and LVFS of the CHF group were significantly lower, and AO, LVPW and IVS of the same group were significantly higher compared with the Con group. The above results indicate that ten weeks volume and eight weeks pressure overload produced CHF successfully in the $\mathrm{CHF}$ and $\mathrm{CHF}$-cur groups. Additionally, ten weeks treatment with curcumin significantly alleviated the symptoms and echocardiographic abnormalities in CHF-cur group (Table I).

Curcumin inhibits heart remodeling. CHF is characterized by both cardiac dysfunction and cardiac remodeling (20). The effects of curcumin on cardiac remodeling was evaluated by cardiac morphology, ultrastructure and histology. Heart/body weight ratio of the $\mathrm{CHF}$ group was higher than the Con group, and curcumin treatment decreased the ratio in the $\mathrm{CHF}$-cur group compared with the CHF group (Fig. 1A). H\&E staining demonstrated that average CSA of LV cardiomyocyte of CHF group was higher than those of Con group significantly, and curcumin ameliorated myocardial hypertrophy (Fig. 1B H\&E and C). Compared with the Con group, the ultrastructure of the $\mathrm{CHF}$ group demonstrated disarranged myofibrils, swelled mitochondrial and diminished crista. Curcumin partly alleviated the ultrastructure disorganization in the CHF-cur group (Fig. 1B Ultrastructure). Marked cardiac fibrosis detected by Masson or PSR staining was demonstrated in the CHF group, and curcumin alleviated fibrosis in the CHF-cur group (Fig. 1D MASSON, 1D PSR, 1E and 1F). Higher apoptosis of cardiomyocyte detected by TUNEL assay was demonstrated in the CHF group, and curcumin ameliorated this effect in the CHF-cur group (Fig. 1D TUNEL and G).

Curcumin regulates the expression of certain $\mathrm{CHF}$ molecular biomarkers. Curcumin was demonstrated to improve cardiac function and inhibit heart remodeling. The effects of curcumin on the biomarkers above, including ROS, TNF- $\alpha$, MMP-2 and MMP-9, were investigated. In the sample images of DHE staining, red granules in cytoplasm and cell nucleus indicated positive staining of ROS (Fig. 2A ROS) and yellow granules in cytoplasm indicated the positive staining of the target proteins in the sample images of immunohistochemical staining (Fig. 2A TNF- $\alpha$, 2D MMP-2, 2D MMP-9 and 2G SERCA2a). Quantitative analysis of the DHE staining and immunohistochemical staining indicated that expression of ROS, TNF- $\alpha$, MMP-2 and MMP-9 were increased in the $\mathrm{CHF}$ group compared with the Con group but these proteins were decreased in the CHF-cur group (Fig. 2A-F). Compared 
Table I. Effects of curcumin on cardiac function and morphology.

\begin{tabular}{|c|c|c|c|c|c|c|}
\hline Group & Time & LVEF (\%) & LVFS (\%) & $\mathrm{AO}(\mathrm{mm})$ & LVPW (mm) & IVS (mm) \\
\hline \multirow[t]{2}{*}{ Con } & Pre & $74.2 \pm 3.8$ & $39.9 \pm 3.3$ & $7.1 \pm 0.2$ & $2.1 \pm 0.2$ & $2.1 \pm 0.1$ \\
\hline & Post & $74.0 \pm 3.4^{\mathrm{b}}$ & $39.8 \pm 2.9^{b}$ & $7.1 \pm 0.3^{\mathrm{b}}$ & $2.2 \pm 0.1^{\mathrm{b}}$ & $2.2 \pm 0.2^{\mathrm{b}}$ \\
\hline \multirow[t]{2}{*}{ Con-cur } & Pre & $71.4 \pm 8.9$ & $38.0 \pm 7.4$ & $7.1 \pm 0.6$ & $2.2 \pm 0.4$ & $2.1 \pm 0.3$ \\
\hline & Post & $70.6 \pm 7.2$ & $37.2 \pm 5.6$ & $7.2 \pm 0.5$ & $2.2 \pm 0.5$ & $2.2 \pm 0.3$ \\
\hline \multirow[t]{2}{*}{$\mathrm{CHF}$} & Pre & $72.1 \pm 6.0$ & $38.2 \pm 5.0$ & $7.2 \pm 0.40$ & $2.2 \pm 0.3$ & $2.1 \pm 0.4$ \\
\hline & Post & $32.1 \pm 8.8^{\mathrm{a}, \mathrm{c}}$ & $14.1 \pm 4.5^{\mathrm{a}, \mathrm{c}}$ & $10.3 \pm 1.5^{\mathrm{a}, \mathrm{c}}$ & $3.1 \pm 0.4^{\mathrm{a}, \mathrm{c}}$ & $4.1 \pm 0.8^{\mathrm{a}, \mathrm{c}}$ \\
\hline \multirow[t]{2}{*}{ CHF-cur } & Pre & $70.2 \pm 5.3$ & $36.7 \pm 4.1$ & $7.2 \pm 0.4$ & $2.0 \pm 0.2$ & $2.1 \pm 0.2$ \\
\hline & Post & $48.3 \pm 12.9^{\mathrm{a}, \mathrm{d}}$ & $23.0 \pm 7.2^{\mathrm{a}, \mathrm{d}}$ & $8.7 \pm 0.5^{\mathrm{a}, \mathrm{d}}$ & $2.6 \pm 0.3^{\mathrm{a}, \mathrm{d}}$ & $3.1 \pm 0.3^{\mathrm{a}, \mathrm{d}}$ \\
\hline
\end{tabular}

Data are expressed as the mean \pm standard deviation $(\mathrm{n}=10)$. All data are expressed as the mean \pm standard deviation $(\mathrm{n}=10)$. ${ }^{\mathrm{a}} \mathrm{P}<0.05$ postoperative parameters vs. preoperative ones; ${ }^{b} \mathrm{P}<0.05$ vs. postoperative parameters of $\mathrm{CHF}$ group; ${ }^{\mathrm{C}} \mathrm{P}<0.05$ vs. postoperative parameters of CHF-cur group; ${ }^{\mathrm{d}} \mathrm{P}<0.05$ vs. postoperative parameters of Con group. AO, aortic diameter; IVS, interventricular septum thickness; LVEF, left ventricular ejection fraction; LVFS, left ventricular fractional shortening; LVPW, left ventricular posterior wall thickness; Post, postoperative; Pre, preoperative.

A
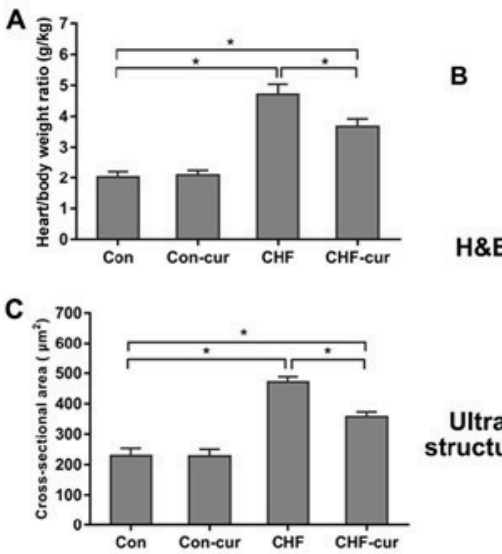

H\&E

Ultrastructure
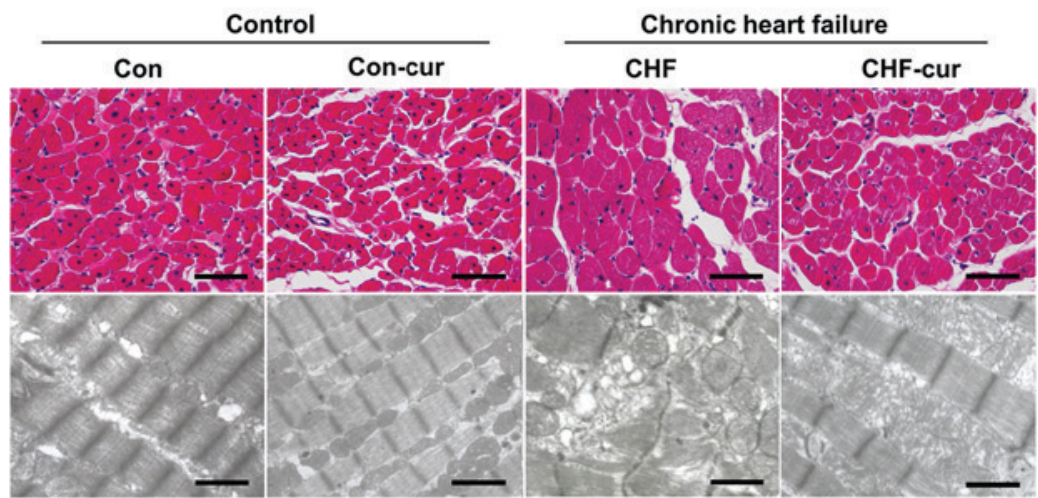

D
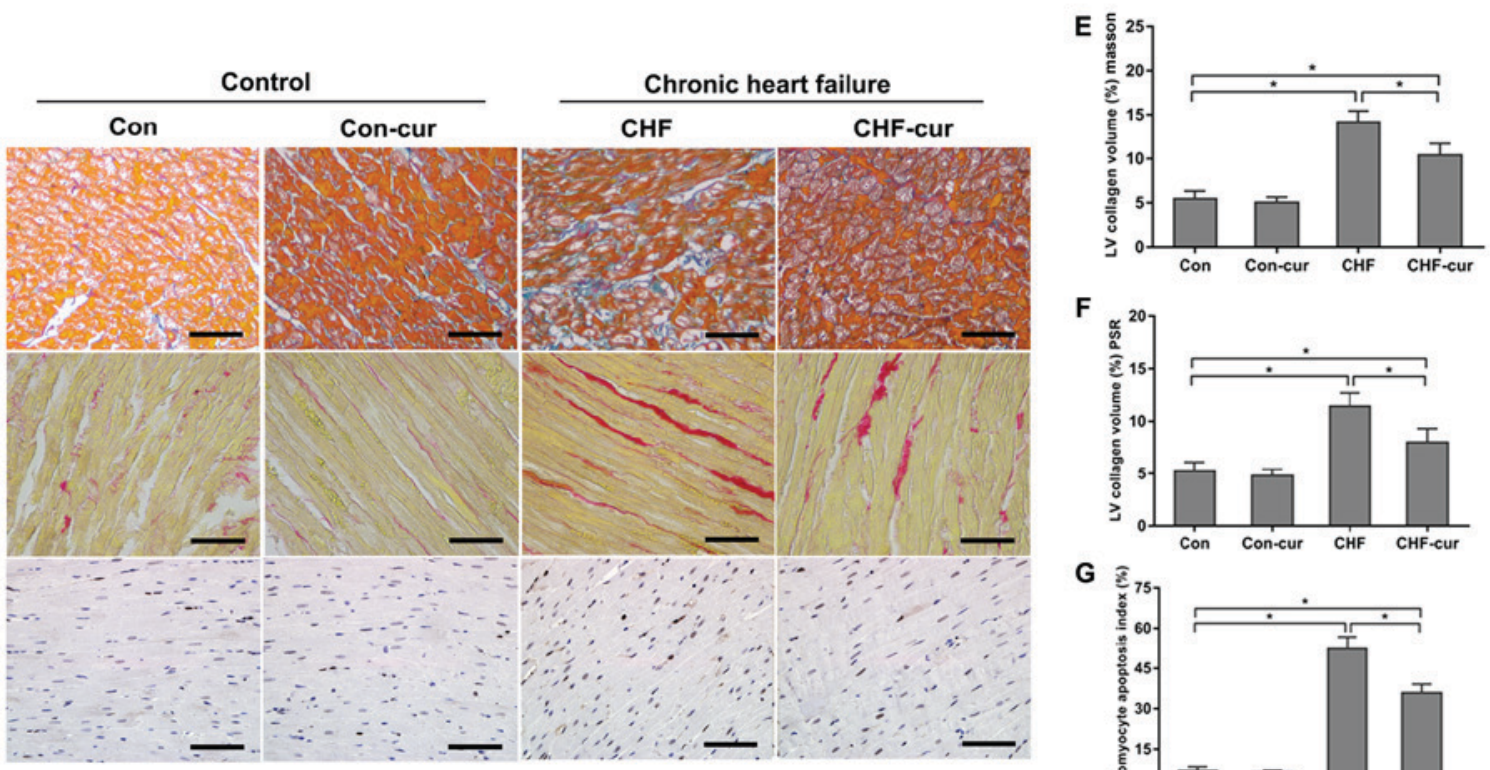

Masson

PSR
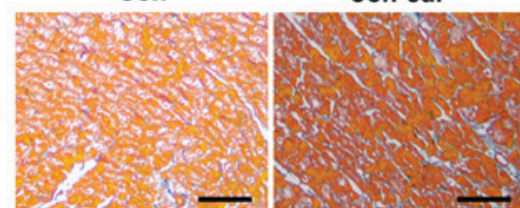

TUNEL
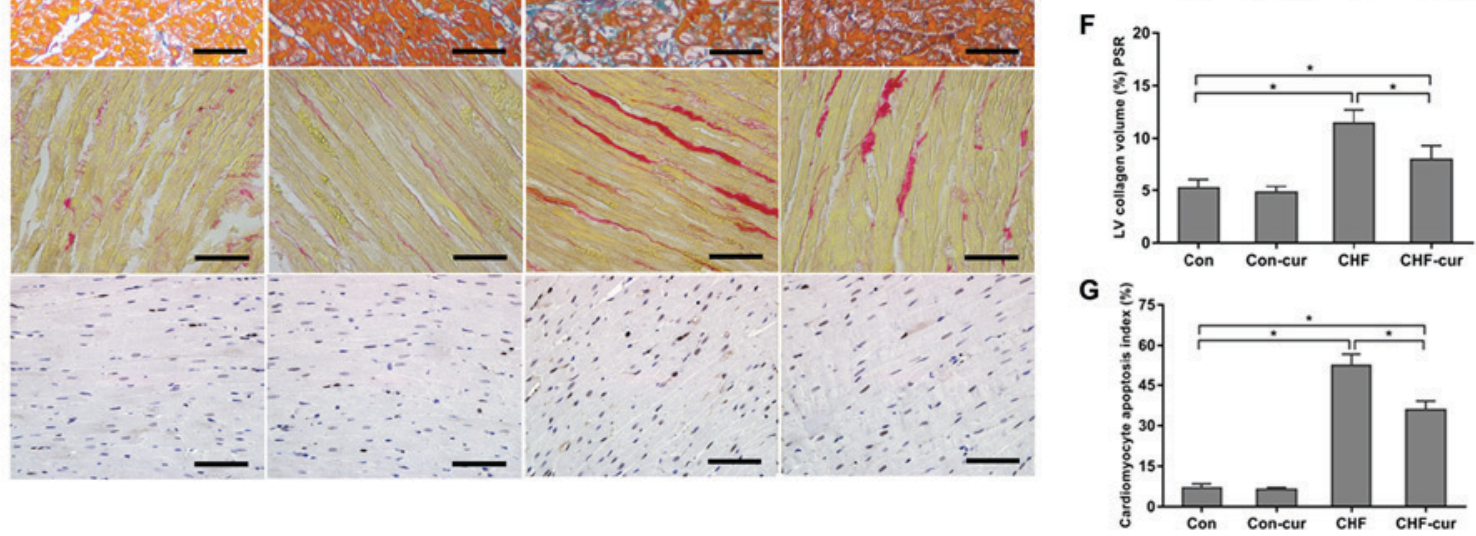

Figure 1. Effects of curcumin on cardiac morphology and histology. (A) Effects of curcumin on heart/body weight ratio. (B) Representative images of H\&E staining (magnification, $\mathrm{x} 200$; scale bar=50 $\mu \mathrm{m}$ ) and ultrastructure of LVAW cardiomyocytes (magnification, $\mathrm{x} 2,000$; scale bar=3 $\mu \mathrm{m}$ ). (C) Effects of curcumin on LVAW cardiomyocytes cross-sectional area. (D) Representative images of LVAW, measured by Masson, picrosirius red staining and TUNEL assay (magnification, $x 200$; scale bar=50 $\mu \mathrm{m}$ ). Quantification of LVAW collagen volume (\%) detected by (E) masson staining, (F) picrosirius red staining. (G) Quantification of cardiomyocyte apoptosis index detected by TUNEL assay. All data are expressed as the mean \pm stadard deviation, $n=10$. "P $<0.05$. CHF, chronic heart failure; Con, control; Cur, curcumin; LVAW, left ventricular posterior wall thickness; TUNEL, terminal deoxynucleotidyl transferase-mediated dUTP nick end labeling; H\&E, haematoxylin \& eosin. 
A

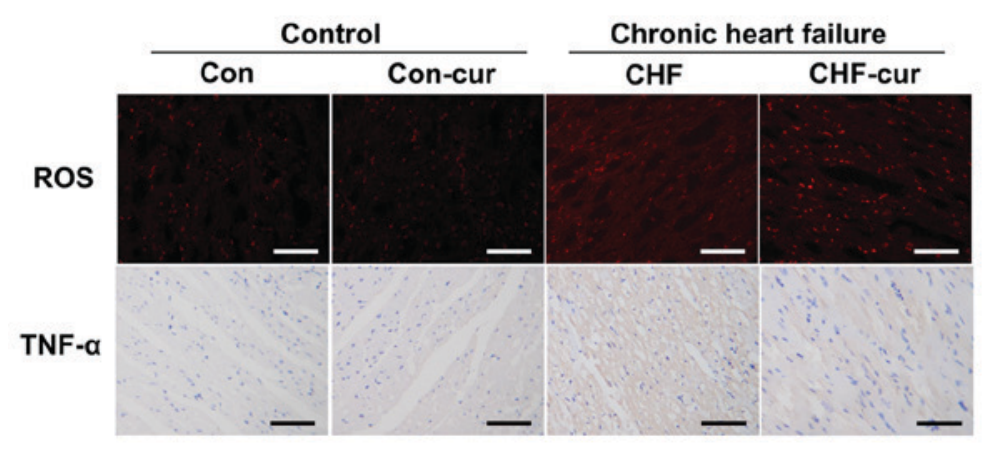

D

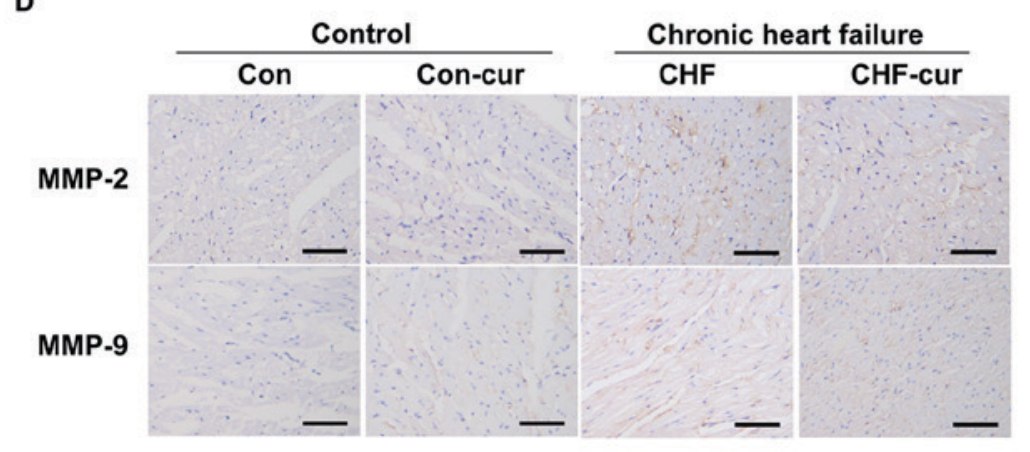

G

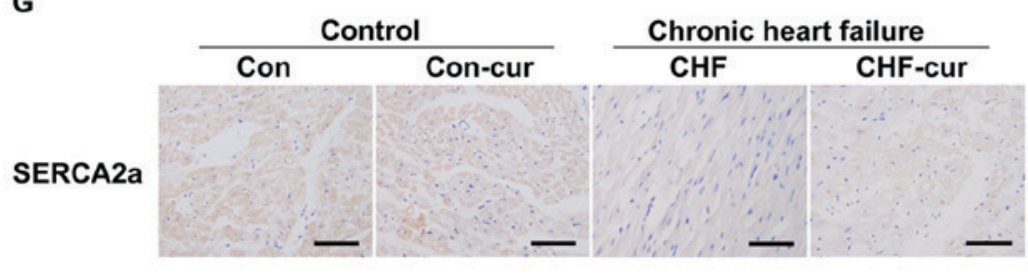

B

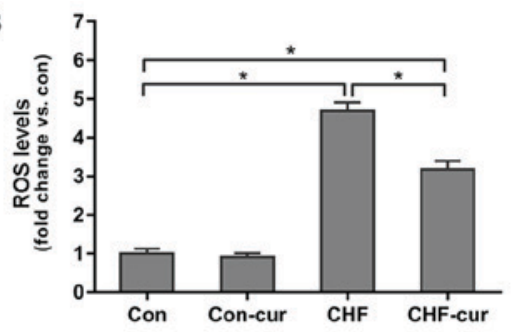

C

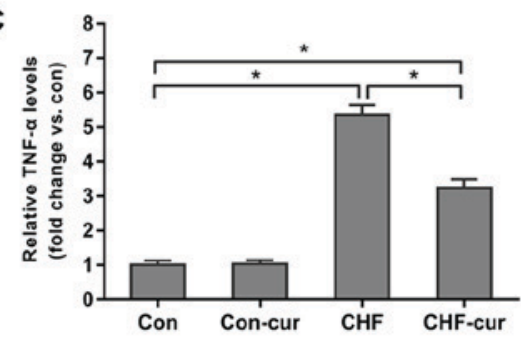

E

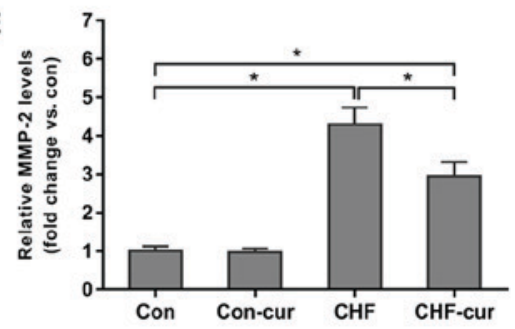

F

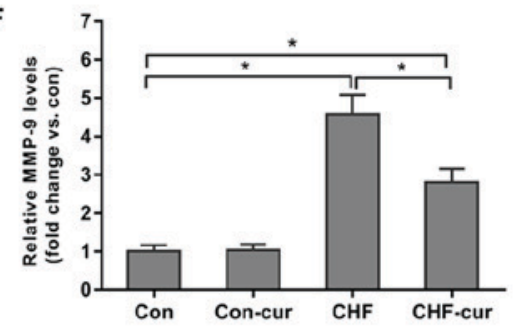

H

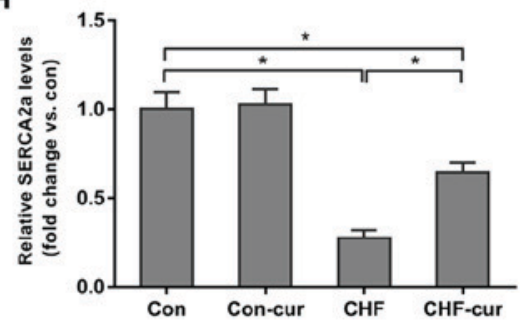

Figure 2. Effects of curcumin on the expression of CHF molecular biomarkers. (A) Representative images of ROS and TNF- $\alpha$ staining and quantification of (B) ROS and of (C) TNF- $\alpha$ expression levels, as measured by DHE (ROS) and immunohistochemical staining (TNF- $\alpha$ ) in LVAW cardiomyocytes. (D) Representative images of MMP-2 and MMP-9 and quantification of (E) MMP-2 and (F) MMP-9 expression levels, as measured by immunohistochemical staining in LVAW cardiomyocytes. (G) Representative images of SERCA2a and (H) quantification of its expression levels, as measured by immunohistochemical staining in LVAW cardiomyocytes. Magnification, $\mathrm{x} 200$. Scale bar $=50 \mu \mathrm{m}$. All data are expressed as the mean \pm stadard deviation, $\mathrm{n}=10$. $\mathrm{P}<0.05$. CHF, chronic heart failure; Con, control; Cur, curcumin; LVAW, left ventricular posterior wall thickness; MMP, matrix metalloproteinase; ROS, reactive oxygen species; SERCA, sarcoplasmic reticulum $\mathrm{Ca}^{2+}$ ATPase; TNF, Tumor necrosis factor; DHE, dihydroethidium.

with the Con group, the expression of SERCA2a decreased in the CHF group, and curcumin increased its expression in the CHF-cur group (Fig. 2G and H).

Curcumin increases expression of DKK-3. Previous studies demonstrated that DKK-3 down-regulation contributed to the stress-induced or infarction-induced dysfunction and remodeling in CHF $(5,16)$. The influence of curcumin on DKK-3 was further investigated. In the sample images of the immunohistochemical staining (Fig. 3A), yellow granules in cytoplasm indicated the expression of DKK-3. As illustrated in the images (Fig. 3A), DKK-3 was mostly expressed in cytoplasm and its expression was higher in the Con group compared with in the CHF group and CHF-cur group. Quantitative analysis of relative DKK-3 expression levels based on immunohistochemical staining indicated that DKK-3 was reduced in the 
A

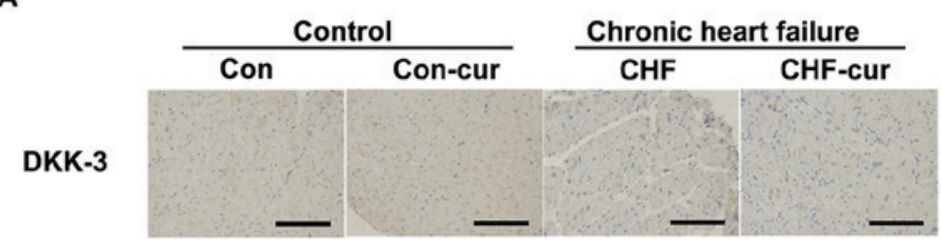

C

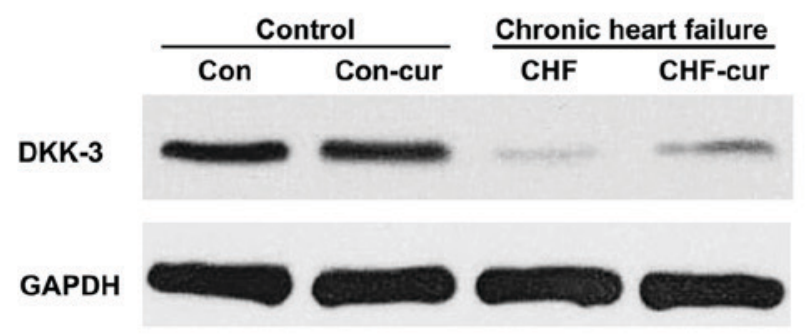

\section{E}
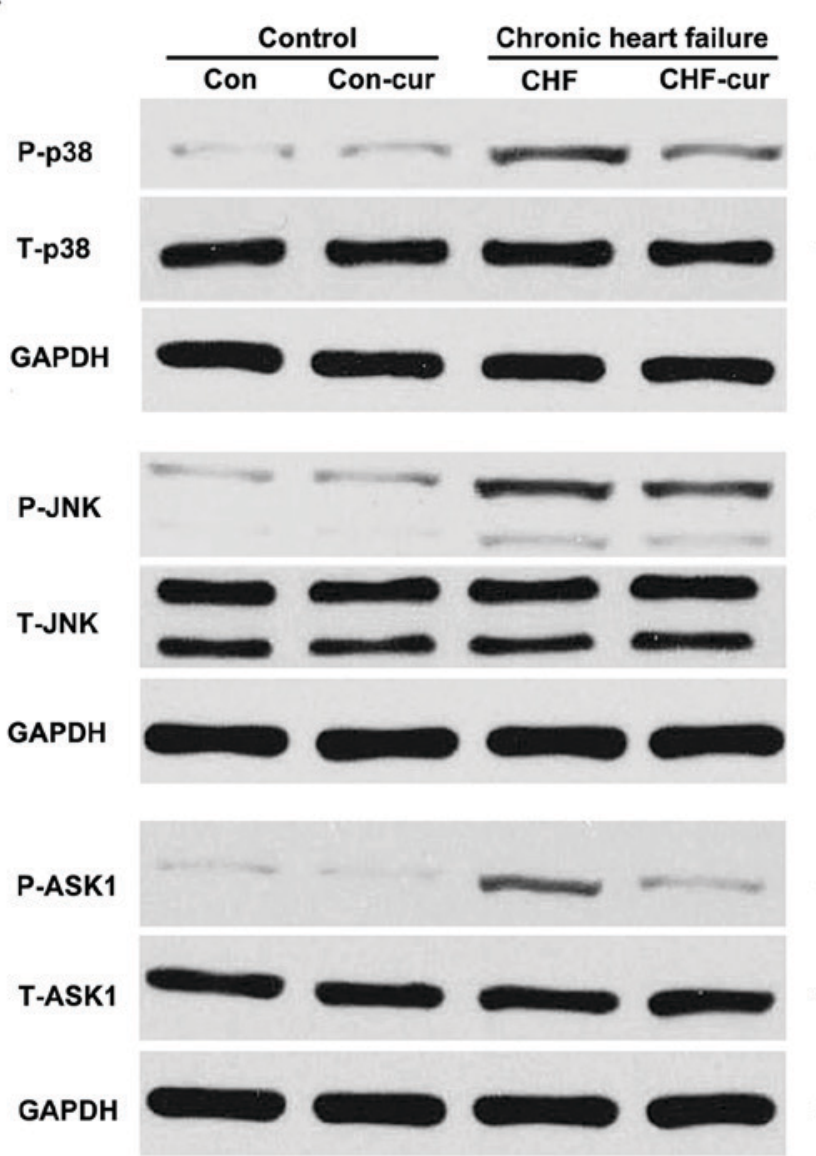

$38 \mathrm{kDa}$

$36 \mathrm{kDa}$
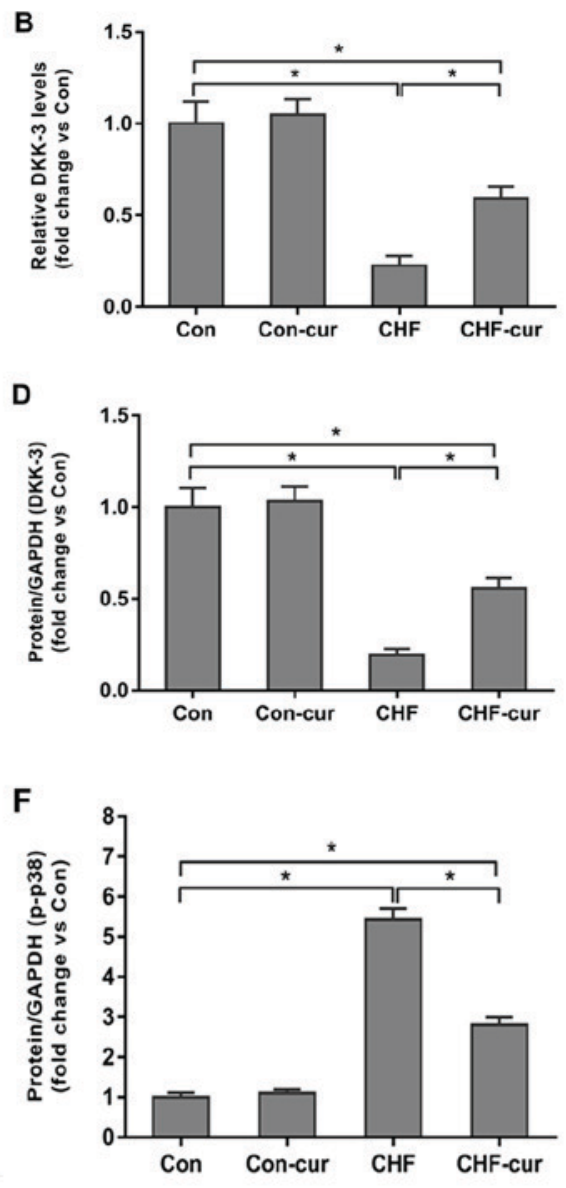

$36 \mathrm{kDa}$

$54 / 46 \mathrm{kDa}$
$54 / 46 \mathrm{kDa}$
$36 \mathrm{kDa}$

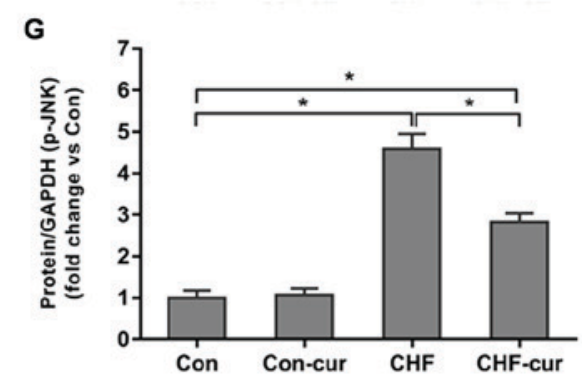

$155 \mathrm{kDa}$

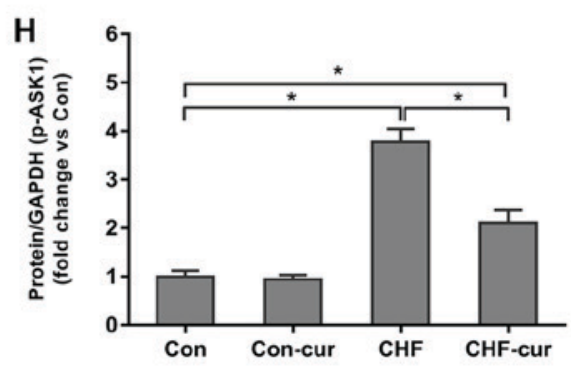

Figure 3. Effects of curcumin on DKK-3, p38, JNK and ASK1 protein expression levels. (A) Representative images of DKK-3 and (B) quantification of its expression levels in LVAW, as evaluated by immunohistochemical staining. (C) Representative images of DKK-3 and (D) quantification of its expression, as evaluated by western blot analysis. (E) Representative images of p-p38, p-JNK and p-ASK1 and quantification of (F) p-p38; (G) p-JNK and (H) p-ASK1 expression levels, as measured by western blot analysis. Magnification, $\mathrm{x} 200$. Scale bar=50 $\mu \mathrm{m}$. All data are expressed as the mean \pm stadard deviation, $\mathrm{n}=10$. ${ }^{*} \mathrm{P}<0.05$. ASK1, apoptosis signal-regulating kinase 1; CHF, chronic heart failure; Con, control; Cur, curcumin; DKK-3, Dickkopf-related protein 3; LVAW, left ventricular posterior wall thickness; $p$, phosphorylated; T, total; p38, p38 mitogen-activated protein kinase; JNK, c-Jun N-terminal kinase.

CHF group compared with the Con group but it was increased in the CHF-cur group (Fig. 3A and B). The western blot analysis indicated that expression levels of DKK-3 were lower in the CHF compared with the Con group, while these were partially restored by curcumin treatment in CHF-cur group
(Fig. 3C and D), which were in accordance to the immunohistochemistry staining results.

Dickkopf-3 upregulation is involved in the cardioprotective effects of curcumin on CHF rabbits. DKK-3 was demonstrated 
to be an inhibitor of p38 and JNK signaling pathways $(5,16)$. In order to reveal the signaling mechanisms involved in the regulation of curcumin, the influence of curcumin on DKK-3, p38 and JNK was investigated. Compared with the Con group, p-p38 and P-JNK were significantly higher in the CHF group. Curcumin decreased p-p38 and P-JNK expression levels in CHF-cur group compared with the CHF group (Fig. 3E-G). This indicated that p38 and JNK signaling pathways were significantly activated during CHF and curcumin partially inhibited the p38 and JNK signaling pathways activation in the CHF-cur group. In addition, ASK1 is regarded as the upstream component of the p38 and JNK signaling pathways and ASK1 phosphorylation is largely responsible for the increase of $\mathrm{p} 38$ and JNK phosphorylation (16). A previous study reported that DKK-3 could protect against cardiac remodeling induced by myocardial infarction or pressure overload via negatively regulating the p38 and JNK signaling pathways in an ASK1-dependent way $(5,16)$. Therefore, the effects of curcumin on ASK1 were further investigated. Corresponding to the p38 and JNK activation, ASK1 was activated in CHF and curcumin partly inhibited its activation in CHF-cur group (Fig. 3E and H).

\section{Discussion}

Rabbit and human hearts are anatomically and functionally similar, and therefore CHF is commonly modeled in rabbits $(18,21)$. Curcumin is a potential candidate for a novel anti-cancer drug and the cardioprotective effects of curcumin on heart diseases have been reported, but the underlying mechanisms have not been revealed fully (9). In the present study, a CHF model was successfully induced by ten weeks volume and eight weeks pressure overload. Ten weeks treatment with curcumin improved cardiac performance, which was indicated by the heart/body weight ratio and echocardiography parameters. Cardiac remodeling was also alleviated by curcumin treatment in the CHF-cur rabbits, including myocardial hypertrophy, fibrosis, apoptosis and ultrastructure disorganization. Curcumin also decreased the expression of some CHF molecular biomarkers. The present study demonstrated that curcumin upregulated DKK-3 expression which may lead to inhibition of p38 and JNK signaling pathways and this may be a potential underlying mechanism by which curcumin exerted cardiac protective effects, but this needs to be confirmed in future studies.

In previous studies, curcumin was reported to exert anti-inflammatory and anti-oxidant effects on several kinds of diseases $(9,22)$. Cardiac remodeling, including cardiac hypertrophy, fibrosis and apoptosis, is partially attributable to overactive inflammatory response and excessive ROS release in CHF (6). When heart suffers from prolonged pressure overload or myocardial infarction, intracellular ROS can activate multiple signaling pathways, including p38 and JNK signaling pathways which may upregulate the expression of TNF- $\alpha$ in myocytes (23). TNF- $\alpha$ in turn increases ROS production by uncoupling mitochondrial respiration in a positive feedback mechanism (6). This pathological process may accelerate the cardiac remodeling through multifaceted impaction $(24,25)$. DKK-3 was also demonstrated to suppress inflammation following myocardial infarction and promote cell survival by reducing the expression of the superoxide during oxidative stress, but the effects of curcumin on DKK-3 has not been clarified $(16,26)$. The present study demonstrated that curcumin increased DKK-3 expression and the increased DKK-3 suppressed inflammatory reactions, and reduced ROS via inhibiting $\mathrm{p} 38$ and JNK signaling pathways in rabbits with CHF.

Curcumin has been reported to serve therapeutic roles on renal and hepatic fibrosis, but rarely on cardiac fibrosis (27-29). An excessive deposition of extracellular matrix (ECM) molecules is another determining factor for cardiac remodeling, especially for myocardial fibrosis $(30,31)$. Previous studies demonstrated that the deposition of ECM positively associates with MMP-2 and MMP-9 levels while tissue inhibitors of metalloproteinases alleviate this deposition $(31,32)$. Upregulation of MMP-2 and MMP-9 in cardiac fibroblasts is associated with the activation of p38 and JNK signaling pathways $(33,34)$. Inducing excessive deposition of ECM, p38 and JNK signaling pathways activation accelerates cardiac fibrosis by promoting migration of inflammatory cells and vascular regeneration through upregulating MMPs (35). A previous study reported that decreased DKK-3 expression leads to up-regulation of MMPs in normal prostate epithelial cells and acts as an inhibitor of p38 and JNK signaling pathways (36). The present study demonstrated that curcumin increased DKK-3 expression and downregulated MMP-2 and MMP-9 possibly by inhibiting p38 and JNK pathways in CHF rabbits.

It has been previously demonstrated that $\mathrm{CHF}$ is characterized by dysregulation of SERCA2a, the major $\mathrm{Ca}^{2+}$ pump in the cardiomyocytes $(37,38)$. SERCA2a can regulate excitation-contraction coupling in cardiomyocytes $(38,39)$. SERCA2a can be regulated precisely according to the physiological condition of the heart $(40,41)$. SERCA2a was identified to be lower in cardiomyocytes of CHF models and an increase of SERCA2a expression could improve cardiac function $(42,43)$. SERCA2a expression was demonstrated to be downregulated by activated p38 and JNK signaling pathways in cultured neonatal and mature cardiomyocytes $(44,45)$. The association between DKK-3 and SERCA2a, as well as the effects of curcumin on SERCA2a, have rarely been studied (46). In the present study, p38 and JNK signaling pathways were inhibited while both DKK-3 and SERCA2a expression levels were upregulated in the CHF-cur group compared with the CHF group. This indicates that curcumin enhanced DKK-3 expression, which in turn may up-regulate SERCA2a via inhibition of p38 and JNK signaling pathways in $\mathrm{CHF}$ rabbits.

It was reported that curcumin exerts anti-inflammatory effects via inhibition of the p38 and JNK pathways in HaCaT cells (47). Activation of p38 and JNK pathways is also involved in the pathology of CHF $(33,48)$. In addition, ASK1 phosphorylation is mainly responsible for the activation of p38 and JNK pathways, and a previous study demonstrated that DKK-3 inhibited them in an ASK1-dependent manner (16). Cardiac hypertrophy and fibrosis were exacerbated by DKK-3 knockout while ameliorated by DKK-3 overexpression (5). In the present study, p38, JNK and ASK1 kinases were all activated in the $\mathrm{CHF}$ group, accompanied by the downregulation of DKK-3 and were partially restored by curcumin in the CHF-cur group 
compared with the CHF group. Yao et al (17) reported that curcumin may improve left ventricular function and remodeling in pressure overloaded rabbits by suppressing myocardial expression of TNF-a and MMP-2. Jeong et al (49) demonstrated that curcumin could attenuate cardiac remodeling in myocardial ischemia/reperfusion (I/R) injury by inhibiting $\mathrm{p} 38$ and JNK pathways in vivo. In addition, curcumin pretreatment was also demonstrated to protect cardiac cells against I/R injury by reducing oxidative stress and inhibiting the phosphorylation of JNK in vitro, as demonstrated by Fiorillo et al (50). The present results are in accordance with previous studies and could serve as additional evidence for the cardioprotective potentials of curcumin in cardiac disease. Furthermore, a potential underlying mechanism was revealed in which curcumin may inhibit p38 and JNK signaling pathways in an ASK1-dependent way via up-regulation of DKK-3 in a rabbit model of CHF. However, there are limitations in the present study for identifying whether knock-out of the DKK-3 gene affects the cardioprotective effect of curcumin and it would be worth further investigation by future studies. The present study proved that DKK-3 upregulation mediates the cardioprotective effects of curcumin on chronic heart failure. Further studies should verify whether knock-out of the DKK-3 gene affects the cardioprotective effect of curcumin and overexpression experiments are needed in order to validate the present findings. Further studies with cardiospecific DKK-3 knockout and overexpression experiments are needed in order to validate the present findings.

\section{Acknowledgements}

Not applicable.

\section{Funding}

The present study was supported by National Natural Science Foundation of China (grant. nos. 81570460, 81500654, 81170767, 81571376 and 81500668); Natural Science Foundation of Hubei Province (grant. no. 2016CFB392); Hubei Province health and family planning scientific research project (grant. no. WJ2017Z003).

\section{Availability of data and materials}

The analyzed data sets generated during the study are available from the corresponding author on reasonable request.

\section{Author's contributions}

QC designed the study and drafted this manuscript; MB directed the design and implementation of this research and is the guarantor of the manuscript; JZ and LG participated in the design of this study, and reviewed and edited this manuscript; YZ and MD collected the relative data and were responsible for the data integrity. All authors approved the final version to be published.

\section{Ethics approval and consent to participate}

All procedures were according to the "Guide for the Care and Use of Laboratory Animals' published by the US National
Institutes of Health (NIH Publication no. 85-23, revised 1996) and approved by the Institutional Animal Care and Use Committee at Renmin Hospital of Wuhan University (Wuhan, China).

\section{Consent for publication}

Not applicable.

\section{Competing interests}

Authors declare they have no competing interests.

\section{References}

1. Cook C, Cole G, Asaria P, Jabbour R and Francis DP: The annual global economic burden of heart failure. Int J Cardiol 171: 368-376, 2014

2. Benjamin EJ, Blaha MJ, Chiuve SE, Cushman M, Das SR, Deo R, de Ferranti SD, Floyd J, Fornage M, Gillespie C, et al: Heart disease and stroke statistics-2017 update: A report from the american heart association. Circulation 135: e146-e603, 2017.

3. Josiak K, Jankowska EA, Piepoli MF, Banasiak W and Ponikowski P: Skeletal myopathy in patients with chronic heart failure: Significance of anabolic-androgenic hormones. J Cachexia Sarcopenia Muscle 5: 287-296, 2014.

4. Koitabashi $\mathrm{N}$ and Kass DA: Reverse remodeling in heart failure-mechanisms and therapeutic opportunities. Nat Rev Cardiol 9: 147-157, 2012.

5. Zhang Y, Liu Y, Zhu XH, Zhang XD, Jiang DS, Bian ZY, Zhang XF, Chen K, Wei X, Gao L, et al: Dickkopf-3 attenuates pressure overload-induced cardiac remodelling. Cardiovasc Res 102: 35-45, 2014.

6. Hofmann U and Frantz S: How can we cure a heart 'in flame'? A translational view on inflammation in heart failure. Basic Res Cardiol 108: 356, 2013.

7. Rahman AF, Angawi RF and Kadi AA: Spatial localisation of curcumin and rapid screening of the chemical compositions of turmeric rhizomes (Curcuma longa Linn.) using direct analysis in real time-mass spectrometry (DART-MS). Food Chem 173: 489-494, 2015.

8. Jankun J, Wyganowska-Swiatkowska M, Dettlaff K, Jelinska A, Surdacka A, Watrobska-Swietlikowska D and Skrzypczak-Jankun E: Determining whether curcumin degradation/condensation is actually bioactivation (Review). Int J Mol Med 37: 1151-1158, 2016.

9. Parada E, Buendia I, Navarro E, Avendano C, Egea J and Lopez MG: Microglial HO-1 induction by curcumin provides antioxidant, antineuroinflammatory, and glioprotective effects. Mol Nutr Food Res 59: 1690-1700, 2015.

10. Zhu X, Li Q, Chang R, Yang D, Song Z, Guo Q and Huang C: Curcumin alleviates neuropathic pain by inhibiting p300/CBP histone acetyltransferase activity-regulated expression of BDNF and cox-2 in a rat model. PLoS One 9: e91303, 2014.

11. Lee JY, Lee YM, Chang GC, Yu SL, Hsieh WY, Chen JJ, Chen HW and Yang PC: Curcumin induces EGFR degradation in lung adenocarcinoma and modulates p38 activation in intestine: The versatile adjuvant for gefitinib therapy. PLoS One 6: e23756, 2011.

12. Morimoto T, Sunagawa Y, Kawamura T, Takaya T, Wada H, Nagasawa A, Komeda M, Fujita M, Shimatsu A, Kita T and Hasegawa K: The dietary compound curcumin inhibits p300 histone acetyltransferase activity and prevents heart failure in rats. J Clin Invest 118: 868-878, 2008.

13. Li HY, Yang M, Li Z and Meng Z: Curcumin inhibits angiotensin II-induced inflammation and proliferation of rat vascular smooth muscle cells by elevating PPAR-gamma activity and reducing oxidative stress. Int J Mol Med 39: 1307-1316, 2017.

14. Ochiai K, Watanabe M, Ueki H, Huang P, Fujii Y, Nasu Y, Noguchi H, Hirata T, Sakaguchi M, Huh NH, et al: Tumor suppressor REIC/Dkk-3 interacts with the dynein light chain, Tctex-1. Biochem Biophys Res Commun 412: 391-395, 2011.

15. Veeck $J$ and Dahl E: Targeting the Wnt pathway in cancer: The emerging role of Dickkopf-3. Biochim Biophys Acta 1825: 18-28, 2012 . 
16. Bao MW, Cai Z, Zhang XJ, Li L, Liu X, Wan N, Hu G, Wan F, Zhang R, Zhu X, et al: Dickkopf-3 protects against cardiac dysfunction and ventricular remodelling following myocardial infarction. Basic Res Cardiol 110: 25, 2015.

17. Yao QH, Wang DQ, Cui CC, Yuan ZY, Chen SB, Yao XW, Wang JK and Lian JF: Curcumin ameliorates left ventricular function in rabbits with pressure overload: Inhibition of the remodeling of the left ventricular collagen network associated with suppression of myocardial tumor necrosis factor-alpha and matrix metalloproteinase-2 expression. Biol Pharm Bull 27: 198-202, 2004

18. Nikolaidou T, Cai XJ, Stephenson RS, Yanni J, Lowe T, Atkinson AJ, Jones CB, Sardar R, Corno AF, Dobrzynski H, et al: Congestive heart failure leads to prolongation of the PR interval and atrioventricular junction enlargement and ion channel remodelling in the rabbit. PLoS One 10: e0141452, 2015.

19. Mukherjee R, Mingoia JT, Bruce JA, Austin JS, Stroud RE, Escobar GP, McClister DM Jr, Allen CM, Alfonso-Jaume MA, Fini ME, et al: Selective spatiotemporal induction of matrix metalloproteinase-2 and matrix metalloproteinase-9 transcription after myocardial infarction. Am J Physiol Heart Circ Physiol 291: H2216-H2228, 2006.

20. Dhalla NS, Saini-Chohan HK, Rodriguez-Leyva D, Elimban V, Dent MR and Tappia PS: Subcellular remodelling may induce cardiac dysfunction in congestive heart failure. Cardiovasc Res 81: 429-438, 2009.

21. Zhang F, Dang Y, Li Y, Hao Q, Li R and Qi X: Cardiac Contractility modulation attenuate myocardial fibrosis by inhibiting TGF- $\beta 1 / \mathrm{Smad} 3$ signaling pathway in a rabbit mode of chronic heart failure. Cell Physiol Biochem 39: 294-302, 2016.

22. Soetikno V, Sari FR, Lakshmanan AP, Arumugam S, Harima M, Suzuki K, Kawachi H and Watanabe K: Curcumin alleviates oxidative stress, inflammation, and renal fibrosis in remnant kidney through the Nrf2-keap1 pathway. Mol Nutr Food Res 57: $1649-1659,2013$.

23. Hori $\mathrm{M}$ and Nishida K: Oxidative stress and left ventricular remodelling after myocardial infarction. Cardiovasc Res 81: 457-464, 2009.

24. Munzel T, Gori T, Keaney JF Jr, Maack C and Daiber A Pathophysiological role of oxidative stress in systolic and diastolic heart failure and its therapeutic implications. Eur Heart J 36: 2555-2564, 2015.

25. Deo SH, Fisher JP, Vianna LC, Kim A, Chockalingam A, Zimmerman MC, Zucker IH and Fadel PJ: Statin therapy lowers muscle sympathetic nerve activity and oxidative stress in patients with heart failure. Am J Physiol Heart Circ Physiol 303: H377-H385, 2012.

26. Qui S, Kano J and Noguchi M: Dickkopf 3 attenuates xanthine dehydrogenase expression to prevent oxidative stress-induced apoptosis. Genes Cells 22: 406-417, 2017.

27. Zhou X, Zhang J, Xu C and Wang W: Curcumin ameliorates renal fibrosis by inhibiting local fibroblast proliferation and extracellular matrix deposition. J Pharmacol Sci 126: 344-350, 2014.

28. Zhang F, Zhang Z, Chen L, Kong D, Zhang X, Lu C, Lu Y and Zheng S: Curcumin attenuates angiogenesis in liver fibrosis and inhibits angiogenic properties of hepatic stellate cells. J Cell Mol Med 18: 1392-1406, 2014

29. Wang ME, Chen YC, Chen IS, Hsieh SC, Chen SS and Chiu $\mathrm{CH}$ : Curcumin protects against thioacetamide-induced hepatic fibrosis by attenuating the inflammatory response and inducing apoptosis of damaged hepatocytes. J Nutr Biochem 23 1352-1366, 2012

30. Bonnans C, Chou J and Werb Z: Remodelling the extracellular matrix in development and disease. Nat Rev Mol Cell Biol 15: 786-801, 2014

31. Vanhoutte D, van Almen GC, Van Aelst LN, Van Cleemput J, Droogne W, Jin Y, Van de Werf F, Carmeliet P, Vanhaecke J, Papageorgiou AP and Heymans S: Matricellular proteins and matrix metalloproteinases mark the inflammatory and fibrotic response in human cardiac allograft rejection. Eur Heart J 34: 1930-1941, 2013.

32. Takawale A, Sakamuri SS and Kassiri Z: Extracellular matrix communication and turnover in cardiac physiology and pathology. Compr Physiol 5: 687-719, 2015.
33. Dodd T, Jadhav R, Wiggins L, Stewart J, Smith E, Russell JC and Rocic P: MMPs 2 and 9 are essential for coronary collateral growth and are prominently regulated by p38 MAPK. J Mol Cell Cardiol 51: 1015-1025, 2011.

34. Liang KC, Lee CW, Lin WN, Lin CC, Wu CB, Luo SF and Yang CM: Interleukin-1beta induces MMP-9 expression via p42/p44 MAPK, p38 MAPK, JNK, and nuclear factor-kappaB signaling pathways in human tracheal smooth muscle cells. J Cell Physiol 211: 759-770, 2007.

35. Cuenda A and Rousseau S: p38 MAP-kinases pathway regulation, function and role in human diseases. Biochim Biophys Acta 1773: 1358-1375, 2007.

36. Romero D, Al-Shareef Z, Gorrono-Etxebarria I, Atkins S, Turrell F, Chhetri J, Bengoa-Vergniory N, Zenzmaier C, Berger P, Waxman J and Kypta R: Dickkopf-3 regulates prostate epithelial cell acinar morphogenesis and prostate cancer cell invasion by limiting TGF- $\beta$-dependent activation of matrix metalloproteases. Carcinogenesis 37: 18-29, 2016.

37. Brini $\mathrm{M}$ and Carafoli E: Calcium pumps in health and disease. Physiol Rev 89: 1341-1378, 2009.

38. Roe AT, Frisk M and Louch WE: Targeting cardiomyocyte $\mathrm{Ca} 2+$ homeostasis in heart failure. Curr Pharm Des 21: 431-448, 2015.

39. Inesi G, Prasad AM and Pilankatta R: The Ca2+ ATPase of cardiac sarcoplasmic reticulum: Physiological role and relevance to diseases. Biochem Biophys Res Commun 369: 182-187, 2008.

40. Cutler MJ, Wan X, Plummer BN, Liu H, Deschenes I, Laurita KR, Hajjar RJ and Rosenbaum DS: Targeted sarcoplasmic reticulum $\mathrm{Ca} 2+$ ATPase 2a gene delivery to restore electrical stability in the failing heart. Circulation 126: 2095-2104, 2012.

41. Huang CL: SERCA2a stimulation by istaroxime: A novel mechanism of action with translational implications. Br J Pharmacol 170: 486-488, 2013.

42. Butter C, Rastogi S, Minden HH, Meyhofer J, Burkhoff D and Sabbah HN: Cardiac contractility modulation electrical signals improve myocardial gene expression in patients with heart failure. J Am Coll Cardiol 51: 1784-1789, 2008

43. Gwathmey JK, Yerevanian A and Hajjar RJ: Targeting sarcoplasmic reticulum calcium ATPase by gene therapy. Hum Gene Ther 24: 937-947, 2013

44. Scharf M, Neef S, Freund R, Geers-Knorr C, Franz-Wachtel M, Brandis A, Krone D, Schneider H, Groos S, Menon MB, et al: Mitogen-activated protein kinase-activated protein kinases 2 and 3 regulate SERCA2a expression and fiber type composition to modulate skeletal muscle and cardiomyocyte function. Mol Cell Biol 33: 2586-2602, 2013

45. Ritchie MF, Zhou Y and Soboloff J: Transcriptional mechanisms regulating $\mathrm{Ca}(2+)$ homeostasis. Cell Calcium 49: 314-321, 2011

46. Sumbilla C, Lewis D, Hammerschmidt T and Inesi G: The slippage of the $\mathrm{Ca} 2+$ pump and its control by anions and curcumin in skeletal and cardiac sarcoplasmic reticulum. J Biol Chem 277: 13900-13906, 2002.

47. Cho JW, Lee KS and Kim CW: Curcumin attenuates the expression of IL-1beta, IL-6, and TNF-alpha as well as cyclin $\mathrm{E}$ in TNF-alpha-treated HaCaT cells; NF-kappaB and MAPKs as potential upstream targets. Int J Mol Med 19: 469-474, 2007.

48. Kaikkonen L, Magga J, Ronkainen VP, Koivisto E, Perjes A, Chuprun JK, Vinge LE, Kilpio T, Aro J, Ulvila J, et al: p38 $\alpha$ regulates SERCA2a function. J Mol Cell Cardiol 67: 86-93, 2014.

49. Jeong CW, Yoo KY, Lee SH, Jeong HJ, Lee CS and Kim SJ: Curcumin protects against regional myocardial ischemia/reperfusion injury through activation of RISK/GSK-3 $\beta$ and inhibition of p38 MAPK and JNK. J Cardiovasc Pharmacol Ther 17: 387-394, 2012.

50. Fiorillo C, Becatti M, Pensalfini A, Cecchi C, Lanzilao L, Donzelli G, Nassi N, Giannini L, Borchi E and Nassi P: Curcumin protects cardiac cells against ischemia-reperfusion injury: Effects on oxidative stress, NF-kappaB, and JNK pathways. Free Radic Biol Med 45: 839-846, 2008.

This work is licensed under a Creative Commons Attribution-NonCommercial-NoDerivatives 4.0 International (CC BY-NC-ND 4.0) License. 